\title{
Consumo abusivo de alcohol en adolescentes españoles: tendencias emergentes y percepciones de riesgo*
}

\author{
Improper Consumption of Alcohol in Spanish Teenagers: \\ Emergent Trends and Perceptions of Risk
}

\author{
MARÍA de la Villa Moral JimÉnEZ ** \\ Departamento de Psicología, Área de Psicología Social, \\ Universidad de Oviedo, España \\ AnAstasio Ovejero Bernal ${ }^{* * *}$ \\ Universidad de Valladolid, España
}

Para citar este artículo. De la Villa Moral, M., \& Ovejero, A. (2011). Consumo abusivo de alcohol en adolescentes españoles: tendencias emergentes y percepciones de riesgo. Universitas Psychologica, $10(1), 71-87$.

* Artículo de investigación.

** Facultad de Psicología. Plaza de Feijóo, s/n - despacho 211, 33003 Oviedo, España. Teléfono: (98) 5103282 . Fax: (98) 5104144 - (98) 5104141.E-mails: mvilla@ uniovi.es; marivilli@telefonica.net

**** Departamento de Psicología Social. C/Real de Burgos, S/N 47011 Valladolid, España. Tel. 983423000.

E-mail: anastasio@psi.uva.es.

RES UMEN

El consumo juvenil de alcohol representa, en la cultura mediterránea, una práctica caracterizada por usos recreativos, ligada a procesos de interacción grupal e identitarios. Nuestro objetivo es ofrecer un análisis descriptivo y diferencial, por niveles de edad e ingesta realizada, de las tendencias de consumo de alcohol, así como de las percepciones de riesgo y de los motivadores de consumo, en un colectivo de 845 adolescentes escolarizados del Principado de Asturias (España). Se confirman las tendencias emergentes sobre hábitos de consumo de alcohol y los patrones de uso/abuso representativos del estilo de ocio juvenil, así como motivaciones y percepciones de riesgo diferenciales, en virtud de la mentalidad del usuario. Se avanzan líneas prospectivas de actuación basadas en intervenciones psicosociales y sociocomunitarias.

Palabras clave autores

Adolescencia, alcohol, sustancias psicoactivas, motivadores de consumo,

prevención, intervención psicosocial.

Palabras clave descriptores

Psicología del adolescente, adicción alcohólica, sustancias peligrosas.

\section{A B S T R A C T}

In the Mediterranean culture the young consumption of alcohol represents a practice associated with a recreative culture tied to processes of group interaction and identity. Our aim is to offer a descriptive and differential analysis for levels of age and ingestion realized of the trends of consumption of alcohol, as well as the perceptions of risk and motivations of consumption in a group of 845 Spanish teenagers of the Principado de Asturias. The emergent trends are confirmed on habits of consumption of alcohol and the representative bosses of use/abuse of the style of juvenile leisure, as well as motivations and differential perceptions of risk by virtue of the mentality of the user. There are advanced market lines of prevention based on psychosocial interventions and socio community.

Key words authors

Adolescence, Alcohol, Psychoactive Substances, Consumption Motivations, Prevention, Pscychosocial Intervention.

Key words plus

Adolescent Psychology, Drinking of Alcoholic Beverages, Hazardous Substances. 


\section{Introducción}

Desde una perspectiva de análisis psicosocial, el consumo juvenil de alcohol representa, en la cultura mediterránea, una práctica ligada a usos lúdicos y recreativos, con base en los mecanismos de identificación y modelado grupal que, dada la propia idiosincrasia de las prácticas de uso y abuso, presenta manifestaciones de consumo masivo ritualizado en grandes espacios vivenciales designados bajo la etiqueta de "botellón" (Aguilera, 2002; Calafat et al., 2004; Cortés, Espejo \& Giménez, 2008; Franco et al., 2005; Gómez-Fraguela, Fernández, Romero $\&$ Luengo, 2008) como principal seña identificativa. Tales prácticas representan una tendencia emergente de esparcimiento juvenil en España, principalmente a partir de la segunda mitad de los años noventa, con un trasfondo eminentemente psicosocial, dada la vinculación al grupo de coetáneos y la participación en prácticas ritualizadas de consumo, ligadas a la extensión de una cultura juvenil recreativa en la esfera del ocio y de ocupación del tiempo libre, así como cambios en la funcionalidad de la diversión nocturna con búsqueda de sobrestimulación y el avance de tendencias expansivas de la popularización del consumo de fin de semana (Bellis y Hughes, 2003; Calafat et al., 2000; Moral y Ovejero, 2006; Sanders, 2006), entre otros indicadores básicos. Semejantes usos/ abusos representan un problema sociosanitario, por la modificación del patrón de consumo socializante propio del modelo latino y su derivación hacia un consumo anglosajón con abundantes episodios de embriaguez, provocado por consumos de bebidas de alta graduación con búsqueda del efecto etílico (uso del alcohol puramente instrumental) y/o por motivación desinhibitoria junto a otros morfotipos toxicofílicos (uso del alcohol como fin objetal por sí mismo), que se recogen bajo la etiqueta de Spain is different, en los términos propuestos por Gual (2006). Semejante problemática es uno de los factores más importantes de riesgo sanitario en todo el mundo (Organización Mundial de la Salud, 2005), siendo considerado como el tercer factor de riesgo en los países industrializados y el principal en los países en desarrollo. Es más, de acuerdo con Torres,
Palma, Iannini y Moreno (2006) se considera que en América Latina y del Caribe, el consumo per capita de alcohol es el más alto del mundo no desarrollado, siendo la droga más usada por los adolescentes (95\% de los jóvenes a la edad de 18 años). Tales datos reflejan la misma tendencia que la hallada en el primer estudio comparativo sobre uso de drogas en población escolar secundaria de Argentina, Bolivia, Brasil, Colombia, Chile, Ecuador, Paraguay, Perú y Uruguay, aportados por el Sistema Subregional de Información e Investigación sobre Drogas bajo la coordinación de Lale-Demoz y Cumsille (2006), que recogen los resultados de las correspondientes encuestas en los países citados bajo el auspicio de la Oficina de Naciones Unidas contra las Drogas y el Delito (ONUDD), el Observatorio Interamericano sobre Drogas de la CICAD/OEA y las comisiones gubernamentales encargadas del tema drogas en los países que son parte de este informe.

Atendiendo a criterios tanto etiopatogénicos como nosológicos, el alcoholismo juvenil tiene entidad propia y diferenciable del alcoholismo del adulto. Entre usuarios también se establecen perfiles diferenciales, en función de las razones por las que los jóvenes beben en grupo los fines de semana y según sus hábitos de bebida y, por extensión otros hábitos sociales, familiares, emocionales e incluso medio-ambientales, de acuerdo con tipologías como las de Jung (Valbuena, Aladro y Sáez, 2007) o a las modificaciones constatables en los morfotipos del joven consumidor (compulsivo, toxicofílico, automedicativo, evasivo, deshinbitorio, socializante, sociopático, etc.) asociadas a politoxiconsumos varios (Sirvent, Moral \& Rodríguez, 2007). Tales riesgos se asocian a un cúmulo de factores determinantes y predisponentes reforzados por el beneficio subjetivo que reporta la propia experimentación al adolescente consumidor (placer, desinhibición, integración, evasión, etc.). De este modo, junto a bases neurobiológicas y químicas (Ayesta, 2002), han de tomarse en consideración tanto la multicausalidad como la sinergia de diferentes factores bioquímicos, personales y psicosociales, como eminentemente socioculturales a modo de un continuum etiológico, según la propuesta de Moral, 
Rodríguez y Sirvent (2005). Sin embargo, a pesar de la constatación de los riesgos físicos, psicológicos y sociales del consumo abusivo de alcohol por parte del colectivo infanto-juvenil la estimación del alcohol como sustancia dañina y como droga portera - de acuerdo con la conocida teoría de la escalada de Kandel $(1975,1978)$-, choca frontalmente tanto con las mentalidades del usuario, como con las representaciones sociales sobre la sustancia y la carga política e ideológica que ello comporta. Respecto a la primera cuestión, entre los usuarios se constata una escasa conciencia del daño ocasionado por el alcohol (Bach i Bach, 2000), lo que conlleva a que no posean suficientes percepciones de riesgo, mientras que presentan una problematicidad distorsionada del tema y, por extensión, unas actitudes de permisividad ante el consumo a nivel tanto cognitivo (creencias, creencias, expectativas, percepciones de riesgo, etc.), como afectivo (identificación con los usuarios, agrado o desagrado, etc.) y comportamental (disposición para el consumo e inclinación para la aceptación del hábito alcohólico) (Moral \& Ovejero, 2003). Asimismo, también se constata la acción de otras posibles influencias de modelos sociales que afectan a su percepción de riesgo, tales como los proporcionados principalmente por el grupo de iguales y el mejor amigo, así como por los padres consumidores y a través de diversos modelamientos simbólicos, tal como han evidenciado Espada, Pereira y García-Fernández (2008). La mentalidad del usuario se relaciona con la comisión de sesgos atributivos asociados a la percepción del consumo de alcohol, entre ellos el sesgo de falsa unicidad (Goethals, Messick \& Allison, 1991), que consiste en considerar que cuando nos comportamos bien, estas acciones normativas son llevadas a cabo por un porcentaje muy pequeño de personas, lo que conlleva también la correspondiente percepción de que la comisión de acciones contra-normativas está más extendida de lo que realmente está, lo cual, en el caso de la experimentación con alcohol, supone la estimación de que su consumo es habitual entre los jóvenes (Yubero, Larrañaga, Navarro, Serna \& Martínez, 2005). Asimismo, ello se relaciona con el sesgo de falso consenso (Krueger \&
Clement, 1994; Marks \& Miller, 1987), que consiste en la tendencia a sobrestimar el grado en que las opiniones propias o las conductas indeseables son comunes y compartidas por muchas personas, lo que conlleva la creencia, entre quienes consumen alcohol, de que son muchos los que consumen la misma cantidad o más que ellos y pocos los que no beben (Igartua, 1996), todo lo cual refuerza la propia mentalidad del usuario y la distorsión de las percepciones de riesgo.

Las motivaciones para el consumo de alcohol se hallan asociadas a la preeminencia otorgada a ciertos valores dominantes (hedonía, consumismo, ocio, sobrestimulación, consumismo, etc.) que definen el estilo de vida dominante en la sociedad española contemporánea (Bilbao, 2008; Bouzada, 2001; Brändle, 2007; Comas, 2004). Según los datos de la Delegación del Gobierno para el Plan Nacional sobre Drogas en España en su Informe de Marzo de 2007, dedicado específicamente a la situación del consumo de alcohol en España, se comprueba que la principal motivación de los jóvenes para el consumo, es la hedónica (Les gusta el sabor, $71.0 \%$; Diversión y placer, $63.6 \%$ ) y en menor medida la ligada a la experimentación de nuevas sensaciones (Sentir emociones nuevas, $15.2 \%)$, u otras finalidades asociadas a su uso evasivo o automedicativo (Superar la timidez, 14.6 $\%$; Olvidar los problemas personales, $15.0 \%$ ). De acuerdo con el Observatorio Español sobre Drogas (2002, 2004), a pesar de la estabilización de la prevalencia de consumo, se evidencia un aumento de los consumos problemáticos y de los porcentajes de bebedores de riesgo con incrementos de urgencias hospitalarias relacionadas con el uso tóxicofílico del alcohol. Semejante tendencia de consumo se asocia a la experimentación con otras sustancias psicoactivas, tales como derivados canábicos (Comas, Jiménez, Acero \& Carballo, 2007; Gómez \& Álvarez, 2006; Olivar \& Carrero, 2007; Vázquez $\&$ Becoña, 2000) y psicoestimulantes (Bobes, Bascarán, González \& Sáiz; 2000; Calafat, Juan, Becoña \& Mantecón, 2008; Infante, Barrio \& Martín, 2003; Sáinz et al., 2001) asociados a la cultura lúdica y recreativa juvenil. Junto a tales características autóctonas, parece existir una cierta similitud 
entre los hábitos de experimentación con alcohol y otras drogas (tabaco y derivados canábicos) de los jóvenes españoles y los del colectivo latinoamericano. En este sentido, a partir de la revisión de la literatura latinoamericana sobre el tema, se incide en la tendencia relativa al incremento de consumo de drogas y con edades de inicio cada vez más tempranas, siendo el alcohol la droga legal más consumida y la ilegal, la marihuana. Esta tendencia es aplicable a diversos países latinoamericanos tales como Colombia (Cáceres, Salazar, Varela \& Tovar, 2006; Londoño \& Valencia, 2008; Salazar \& Arrivillaga, 2004; Torres et al., 2006), según los resultados hallados por estamentos institucionales en la República de Colombia, tales como los informes de la Comisión Nacional de Investigación en Drogas (2001), el Ministerio de Educación (2006) o el Ministerio de Protección Social (2003), entre otros, así como en países como México (Examen Médico Automatizado para Estudiantes [EMA], 2005; Herrera-Vázquez, Wagner, Velasco-Mondragón, Borges \& Lazcano-Ponce, 2004; Urquieta, Hernández-Ávila \& Hernández, 2006), Chile (Rodríguez, Valdés, Ramírez, Hernández, Villalón \& Fernández , 2008), Brasil (Bravo, 2007; Lucas, Parente, Picanço, Conceição, Magalhães \& Siqueira, 2005) o el Caribe según datos de la Organización Panamericana de la Salud (2005), entre otros. De este modo, al parecer y según los datos de que disponemos, las pautas de consumo juveniles propias del arco mediterráneo y del hispanoamericano son similares, aunque es necesario profundizar en el estudio de sus características idiosincrásicas.

De acuerdo con lo anterior, el objetivo principal de la investigación se concreta en el análisis de los hábitos de consumo de alcohol, según indicadores tales como la edad de inicio a la experimentación, así como de las percepciones de riesgo asociadas de un colectivo de jóvenes españoles según un autodiagnóstico de consumo por tipo de bebedores (desde excesivos hasta abstemios), proponiéndose un modelo explicativo del uso/abuso del consumo de alcohol. Se plantea la hipótesis relativa a la menor conciencia de nocividad de las prácticas de consumo en adolescentes de mayor nivel de edad, según las percepciones de riesgo distorsionadas por la mentalidad del usuario, así como en quienes reportan un uso abusivo de alcohol en relación con aquellos que consideran que hacen un consumo responsable o no consumen.

\section{Método}

\section{Participantes}

A través de convenios de colaboración con seis Centros de Educación Secundaria del Principado de Asturias (España), se ha efectuado un muestreo intencional por conglomerados, seleccionándose varios grupos escolares de diferentes niveles académicos constituyendo la población muestral potencial todo el colectivo de alumnos adscritos a la Enseñanza Secundaria Obligatoria (ESO) y Bachillerato. La muestra está integrada por 845 sujetos, de los cuales 459 son chicas que representan el 54.3 $\%$ y 386 chicos que conforman el $45.7 \%$ restante, con edades que oscilan entre los 12 y los 18 años, situándose la moda en la edad de 16 años y la media en 15.39 años. De acuerdo con su adscripción a un nivel académico, se constata que la mayoría de los adolescentes cursan niveles superiores de Enseñanza Secundaria Obligatoria ( $4^{\circ}$ y $3{ }^{\circ}$ de ESO) con porcentajes del $26.4 \%(n=223)$ y del 23.7 $\%$ del alumnado $(n=200)$, respectivamente, asî como 1er curso de Bachillerato $(n=215)$. Nuestros sujetos provienen en su mayoría (76.8 \%) de un hogar nuclear, en el cual el nivel cultural paterno y materno es medio, habiendo cursado estudios de Bachillerato Superior o similar el 24.0 \% y el 27.7 $\%$ respectivamente, siendo el $20.9 \%$ hijos únicos. Por lo que respecta a variables pertinentes al objeto de este estudio, un $9.4 \%(n=79)$ reconocen que beben en exceso, un $39.1 \%(n=329)$ que bastante o con moderación, mientras que informan beber poco un $33.1 \%(n=279)$ y se declaran abstemios un $18.4 \%(n=155)$ (véase Tabla 1$)$.

\section{Variables investigadas e instrumento de medida}

Nuestro interés investigador se concreta en el análisis de los hábitos de consumo de alcohol y 
TABLA 1

Análisis descriptivo de la muestra

\begin{tabular}{|c|c|c|}
\hline \multirow{2}{*}{$\begin{array}{l}\text { Distribución muestral } \\
\text { muestra edad/sexo }\end{array}$} & \multicolumn{2}{|c|}{ Muestra total $(n=845)$} \\
\hline & Varón $n \%$ & Mujer $n \%$ \\
\hline 12 Años & 328.78 & 226.0 \\
\hline 13 & 13.0 & 4712.8 \\
\hline 14 & 7921.4 & 9626.2 \\
\hline 15 & 10929.5 & 10127.6 \\
\hline 16 & 6016.3 & 6517.6 \\
\hline 17 & 308.1 & 246.6 \\
\hline 18 & 113.0 & 113.0 \\
\hline \multirow{2}{*}{$\begin{array}{c}\text { Distribución muestral } \\
\text { cursos /sexo }\end{array}$} & \multicolumn{2}{|c|}{ Muestra total $(n=845)$} \\
\hline & Varón $n$ \% & Mujer $n \%$ \\
\hline $1^{\mathrm{o}}$ e.S.O. & 12.5 & 6414.0 \\
\hline $2^{\circ}$ e.S.O. & 4010.4 & 5311.6 \\
\hline 3e e.S.O. & 8121.1 & 11725.7 \\
\hline $4^{\mathrm{o}}$ e.S.O. & 10326.9 & 12026.3 \\
\hline $1^{\mathrm{o}}$ bachiller & 11129.0 & 10222.4 \\
\hline $\begin{array}{c}\text { Nivel cultural paterno y } \\
\text { materno }\end{array}$ & $\begin{array}{l}\text { Paterno } \\
\text { n } \%\end{array}$ & $\begin{array}{l}\text { Materno } \\
\mathrm{n} \%\end{array}$ \\
\hline Sin estudios & 536.3 & 596.9 \\
\hline Primarios completos & 19222.8 & 17420.7 \\
\hline Bachiller elemental & 16219.3 & 18121.5 \\
\hline Bachiller superior & 20224.0 & 23327.7 \\
\hline Universitarios medios & 11213.3 & 12715.2 \\
\hline Universitarios superiores & 12014.3 & 677.9 \\
\hline
\end{tabular}

Fuente: elaboración propia.

de otras sustancias psicoactivas asociados al estilo de ocio juvenil propio de la cultura recreativa. De ahí que se haya procedido a realizar un análisis del hábito social de consumo de alcohol en el último mes (frecuencia de consumo, número de borracheras, experimentación con otras sustancias) y de la percepción del grado de problematicidad derivado del consumo de diversas sustancias psicoactivas. El análisis de los motivadores de consumo de la primera experimentación y sucesivas, así como el hábito social de consumo de alcohol y experimentación con alcohol en el último mes (tipo de bebida y frecuencia de consumo) completa el análisis, al que se suma un análisis diferencial en todos los indicadores citados, en virtud del autodiagnóstico de los propios adolescentes de acuerdo al indicador:
“¿Cuánto dirías que bebes?” con cuatro modalidades de respuesta (En exceso, Bastante, Poco y Nada) en virtud del cual se han establecido conglomerados por ingesta realizada (Uso/abuso de alcohol). Se ha empleado un instrumento construido ad hoc por los investigadores - la Escala de Hábitos y Actitudes de Consumo de Alcohol (Moral et al., 2005) -, integrada por 45 preguntas formuladas con diversas modalidades de respuesta tales como formato Likert de cinco puntos (MD a MA) para la evaluación de las actitudes hacia el consumo de alcohol y evaluación de frecuencias (Nunca a Casi Siempre) de consumo, percepciones de riesgo, hábito lúdico, motivadores de consumo y otros indicadores, mediante los que se exploran prácticas asociadas pertinentes al objeto de estudio. Por lo que respecta a las cualidades psicométricas de la escala, se afirma que es fiable y válida, habiéndose obtenido puntuaciones elevadas $(>$.804) en el indicador de Cronbach prueba de una buena consistencia interna, siendo el valor del coeficiente ALPHA de .84 para el total de ítems que componen el instrumento.

\section{Procedimiento}

Dado el carácter longitudinal de la experiencia, en primera instancia se ha administrado anónimamente (aunque con la adjudicación de un código) la citada Escala de Hábitos y Actitudes de Consumo de Alcohol a la muestra de estudiantes de Secundaria, en un intervalo temporal de cuatro semanas. A partir del análisis epidemiológico de los hábitos de consumo de alcohol y experimentación con otras sustancias psicoactivas, nuestro interés investigador se concreta en el diseño e implementación de estrategias de prevención primaria del alcoholismo juvenil, para lo cual se ha elaborado un Conjunto Didáctio-Audiovisual titulado Jóvenes $\mathbb{F}$ Alcohol (Sirvent et al., 2007) que incluye contenidos teóricos y eminentemente aplicados (DVD, manual de utilización para educadores o técnicos, fichas de trabajo, etc.), de cara a la posterior intervención preventiva, si bien en este artículo se ofrecerán exclusivamente los resultados de la primera encuesta. 


\section{Análisis de datos}

Se ha distribuido la muestra en conglomerados, mediante el procedimiento de extracción de kmedias, proponiendo un número máximo de cuatro conglomerados, lo cual ha permitido configurar la variable "tipo de consumidor" con cuatro niveles. Asimismo, se ha conformado la variable "niveles de edad". Se han realizado diversos análisis de varianza (ANOVA), para comprobar si existen diferencias significativas en los indicadores pertinentes al objeto de estudio. Asimismo, se aporta un listado de porcentajes de respuesta de acuerdo con los niveles explicitados, y se han empleado pruebas de comparaciones de medias con el objeto de comprobar si los diferentes tipos de consumidores difieren en sus hábitos de consumo, en los motivadores que los impulsan a la experimentación y a las percepciones de riesgo asociadas, así como análisis de regresión en el indicador "percepción de uso/abuso de alcohol".

\section{Resultados}

En primera instancia, a partir del análisis descriptivo diferencial de las prácticas de consumo de alcohol por nivel de edad, se ha confirmado que, entre el colectivo juvenil español, la experimentación en espacios vivenciales masificados bajo la modalidad calificada como "botellón", sigue constituyendo una práctica habitual tanto para los adolescentes de 12 a 14 años (42.6\%) como para los del espectro de edad de 15 a 18 (61.3\%), a pesar de las regulaciones legislativas al respecto, en buena parte del territorio nacional español (véase Vega, 2002). Junto a esta práctica, la modalidad de consumo en "bares o pubs con amigos" es la más practicada, especialmente entre el colectivo juvenil de mayor edad ( $80.9 \%$ de acuerdo), si bien es sumamente preocupante que el $51.0 \%$ de los adolescentes de entre 12-14 años reconozcan consumir alcohol en locales en los que legalmente está prohibida la venta de alcohol a menores de 18 años. Por el trasfondo psicosocial y clínico de las experimentaciones en soledad, destacan los porcentajes que rondan el quince por ciento de adolescentes de ambos niveles de edad que reconocen consumir alcohol principalmente en casa (15.5\% y $16.6 \%)$ o fuera de casa solos ( $16.0 \%$ y $15.3 \%)$, ya que este morfotipo de joven consumidor en soledad tiende a presentar mayores desajustes psicopatológicos y relacionales, siendo más proclives al perfil tóxicofílico y evasivo del fracaso personal o ambiente conflictivo (Sirvent et al., 2007). En relación con tipo de bebida consumida por los adolescentes, se confirma la preferencia por el consumo de bebidas de "baja" graduación (calimocho, cerveza y combinados). De acuerdo con los datos recogidos por Rodríguez-Martos (1996), el beber hasta alcanzar el puntillo y de forma concentrada en las noches de fin de semana, aumenta en el período entre 16-20 años, declinando después, a lo que habría que sumar prácticas abusivas de consumo que conducen a un uso toxicofílico del alcohol hasta alcanzar borracheras (Casa, O’Ferral \& Vaca, 2001) (véase Tabla 2).

Según los resultados expuestos en la Tabla 3, que incluyen el autodiagnóstico de uso/abuso de consumo de alcohol en función de la edad de los usuarios, se confirma que los adolescentes de las edades de mayor riesgo para la experimentación y/o consumo abusivo, serían a partir de los 14 años con porcentajes de consumo que superan el $70 \%$. En más, en la muestra de 17 años el $92.8 \%$ reconoce beber ya sea poco $(28.3 \%)$, con moderación (51.1\%) o en exceso (13.3\%). Por su especial significación psicosocial y por las implicaciones psíquicas y de índole metabólico, ha de apuntarse que para la muestra seleccionada de 12 años, un $44.2 \%$ restante reconoce beber ya sea poco (15.8 $\%)$, con moderación (13.2\%) o en exceso (5.3\%). Porcentajes de respuesta indicativos de mayores niveles de experimentación han sido hallados entre los adolescentes de 13 años (nada: $44.6 \%$, poco: $39.2 \%$, con moderación: $10.8 \%$ y en exceso: $5.4 \%)$ e incrementándose en niveles superiores. Tales resultados van en la línea de otros en los que se evalúan los patrones de consumo autoinformados y los umbrales de riesgo para la salud de los adolescentes, como el estudio de Salamó, Gras y Font-Mayolas (2010). 
TABLA 2

Hábito social de consumo de alcohol. Porcentajes de respuesta por niveles de edad

\begin{tabular}{|c|c|c|c|c|c|c|c|c|c|c|}
\hline \multirow{3}{*}{$\begin{array}{l}\text { Hábito social de } \\
\text { consumo de alcohol }\end{array}$} & \multicolumn{10}{|c|}{ Muestra Total $(N=845)$} \\
\hline & \multicolumn{5}{|c|}{$12-15$ años (\%) } & \multicolumn{5}{|c|}{$16-18$ años (\%) } \\
\hline & Nunca & Pocas v. & Bastante & Muchas v. & Siempre & Nunca & Pocas v. & Bastante & Muchas v. & Siempre \\
\hline En casa, yo solo & 85.5 & 8.9 & 2.7 & 1.2 & 1.7 & 83.4 & 10.0 & 3.2 & 2.5 & 0.9 \\
\hline Fuera de casa, yo solo & 84.0 & 10.8 & 2.5 & 1.2 & 1.5 & 84.7 & 8.4 & 2.7 & 2.7 & 1.4 \\
\hline En casa, con mi familia & 78.1 & 13.5 & 4.2 & 2.2 & 2.0 & 74.0 & 18.0 & 4.6 & 2.1 & 11.4 \\
\hline $\begin{array}{l}\text { En mi casa o en la de un } \\
\text { amigo }\end{array}$ & 65.5 & 13.8 & 8.4 & 9.6 & 2.7 & 44.6 & 22.1 & 15.3 & 12.1 & 15.9 \\
\hline $\begin{array}{l}\text { En bares baratos con } \\
\text { amigos }\end{array}$ & 52.7 & 18.0 & 6.2 & 11.3 & 11.8 & 26.9 & 17.1 & 18.5 & 22.3 & 15.3 \\
\hline En bares o pubs con amigos & 49.0 & 14.5 & 8.9 & 16.3 & 11.3 & 19.1 & 15.9 & 21.0 & 26.0 & 18.0 \\
\hline $\begin{array}{l}\text { En la calle con amigos } \\
\text { ("botellón") }\end{array}$ & & 15.0 & & & & & & & & 10.3 \\
\hline En discotecas & 56.7 & 15.5 & 9.4 & 10.3 & 8.1 & 29.2 & 17.8 & 17.1 & 21.2 & 14.8 \\
\hline En otros sitios & 77.8 & 9.6 & 5.2 & 3.7 & 3.7 & 62.4 & 18.7 & 8.0 & 6.4 & 4.6 \\
\hline \multirow{2}{*}{$\begin{array}{l}\text { Consumo de alcohol en } \\
\text { el último mes }\end{array}$} & \multicolumn{5}{|c|}{ 12-14 años (\%) } & \multicolumn{5}{|c|}{ 15-18 años (\%) } \\
\hline & Nunca & Pocas v. & Bastante & Muchas v. & Siempre & Nunca & Pocas v. & Bastante & Muchas v. & Siempre \\
\hline Vino & 74.1 & 15.0 & 5.2 & 2.7 & 3.0 & 69.9 & 14.4 & 8.4 & 3.0 & 4.3 \\
\hline Cerveza & 65.8 & 17.2 & 5.7 & 7.6 & 3.4 & 16.9 & 17.8 & 12.3 & 14.1 & 8.9 \\
\hline Combinados & 62.8 & 14.5 & 7.8 & 9.4 & 5.4 & 42.1 & 15.7 & 15.7 & 12.5 & 13.9 \\
\hline Aperitivos & 69.5 & 16.2 & 5.4 & 5.9 & 3.0 & 55.6 & 20.0 & 11.8 & 8.0 & 4.6 \\
\hline Licores calimocho & 53.0 & 15.5 & 9.9 & 9.9 & 11.8 & 38.7 & 16.9 & 15.0 & 15.0 & 14.4 \\
\hline Otros & 51.2 & 16.0 & 9.9 & 13.3 & 9.6 & 32.9 & 23.3 & 18.9 & 13.2 & 11.6 \\
\hline
\end{tabular}

Fuente: elaboración propia.

TABLA 3

Autodiagnóstico en el consumo de alcohol según la edad de los usuarios

\begin{tabular}{|c|c|c|c|c|c|c|c|c|c|c|}
\hline & & \multicolumn{8}{|c|}{ ¿Cuánto dirías que bebes? } & \multirow{3}{*}{ Total } \\
\hline & & \multicolumn{2}{|c|}{ En exceso } & \multicolumn{2}{|c|}{ Con moderación } & \multicolumn{2}{|c|}{ Poco } & \multicolumn{2}{|c|}{ Nada } & \\
\hline & & $\mathrm{N}$ & $\%$ & $\mathrm{~N}$ & $\%$ & $N$ & $\%$ & $\mathrm{~N}$ & $\%$ & \\
\hline \multirow[t]{7}{*}{ Edad } & 12 & 2 & 5.3 & 5 & 13.2 & 6 & 15.8 & 25 & 65.8 & 38 \\
\hline & 13 & 4 & 5.4 & 8 & 10.8 & 29 & 39.2 & 33 & 44.6 & 74 \\
\hline & 14 & 0 & 0.0 & 32 & 34.0 & 32 & 34.0 & 30 & 31.9 & 94 \\
\hline & 15 & 13 & 6.5 & 80 & 40.2 & 76 & 38.2 & 30 & 15.1 & 199 \\
\hline & 16 & 30 & 13.5 & 92 & 41.3 & 78 & 35.0 & 23 & 10.3 & 223 \\
\hline & 17 & 24 & 13.3 & 92 & 51.1 & 51 & 28.3 & 13 & 7.2 & 180 \\
\hline & 18 & 6 & 17.6 & 20 & 58.8 & 7 & 20.6 & 1 & 2.9 & 34 \\
\hline Total & & 79 & 9.38 & 329 & 39.07 & 279 & 33.13 & 155 & 18.40 & 842 \\
\hline
\end{tabular}

Fuente: elaboración propia. 
A partir del análisis diferencial de los motivadores de consumo en función del consumo percibido de alcohol, se ha hallado una tendencia a incrementarse los motivadores con sustrato clínico de la primera experimentación en adolescentes que consumen abusivamente, tales como el motivador relativo a "olvidar" $\left(F_{(1,844)}=11.279, p<.0001\right)$ $\mathrm{u}$ otros vinculados a aspectos motivacionales tales como "evadir la rutina" $\left(F_{(1,844)}=18.168, p<\right.$ .0001) y deshinbitorios tales como sentir "euforia" $\left(F_{(1,844)}=40.279, p<.0001\right)$ y el deseo de "desinhibirse" $\left(F_{(1,844)}=35.363, p<.0001\right)$. También se ha comprobado la modulación psicosocial del proceso de habituación, ya que entre los consumidores abusivos se valora el modelado de consumo del grupo de iguales ("porque beben mis amigos") $\left(F_{(1,844)}=\right.$ $29.904, p<.0001$ ) (véase Tabla 4).

En la literatura sobre el tema se constata que el grado de riesgo percibido derivado del consumo de drogas no tiende a ajustarse, en sentido restringido, a los daños reales derivados del consumo, de ahí que se aluda al concepto de mentalidad del usuario (Echeburúa, 1995; Echeburúa \& Del Corral, 1988). Según los resultados porcentuales expuestos en la Tabla 5 referentes al acuerdo mostrado ante el grado de problematicidad o riesgo percibido en prácticas de experimentación y habituación a diversas sustancias psicoactivas (tabaco, alcohol, cannabis, tranquilizantes, éxtasis y cocaína), se observan diferencias atribuibles al tipo de bebedor (Excesivo a Abstemio) en el sentido predicho. Esto es, los adolescentes que se declaran como bebedores abusivos presentan porcentajes más bajos de concienciación ante los riesgos en todos los indicadores, lo que es reflejo de una menor percepción de riesgo ante los efectos dañinos potenciales del uso/abuso de las drogas, de acuerdo a la aludida mentalidad del usuario. Semejante tendencia de respuesta se invierte en adolescentes abstemios que son más conscientes de los riesgos potenciales de tales prácticas de consumo y habituación. Con el objeto de determinar la existencia de diferencias significativas atribuibles a las experiencias de uso/abuso, se ha realizado una comparación de medias mediante el procedimiento ANOVA. Se ha comprobado la significación de las diferencias atribuibles al tipo de bebedor en los riesgos percibidos en la dependencia tabáquica $\left(F_{(1,844)}=\right.$ $7.254, p<.05)$, la habituación al alcohol $\left(F_{(1,844)}=\right.$ $4.256, p<.05)$, el consumo habitual de derivados canábicos $\left(F_{(1,844)}=3.841, p<.05\right)$, y el consumo experimental de éxtasis $\left(F_{(1,844)}=4.118, p<.05\right)$ y cocaína $\left(F_{(1,844)}=3.078, p<.05\right)$, mostrando mayor concienciación todos los colectivos respecto a la posible habituación a los tranquilizantes, éxtasis y cocaína (véase Tabla 6).

TABLA 4

Motivadores de consumo. Diferencias por tipos de bebedores

\begin{tabular}{lccccc}
\hline \multirow{2}{*}{\begin{tabular}{c} 
Motivadores de consumo $\begin{array}{c}\text { Primera } \\
\text { Experimentación }\end{array}$ \\
\cline { 2 - 6 } \cline { 2 - 6 }
\end{tabular}} & \multicolumn{5}{c}{ Muestra Total $(\mathrm{N}=845)$} \\
\cline { 2 - 6 } & Bebedores en Exceso & Bastante/ Moderación & Poco & Nada & Tukey-B \\
\hline Olvidar & $42.5(37)^{*}$ & $39.3(128)^{*}$ & $27.4(76)^{*}$ & $12.3(19)^{*}$ & $e>b>p>n$ \\
Evadir la rutina & $49.4(43)^{*}$ & $51.8(169)^{*}$ & $39.0(108)^{*}$ & $14.3(22)^{*}$ & $e>b>p>n$ \\
Beben mis amigos & $63.2(55)^{*}$ & $66.6(217)^{*}$ & $49.8(138)^{*}$ & $16.9(26)^{*}$ & $e, b>p>n$ \\
Me incitaron a ello & $60.8(53)$ & $59.8(195)$ & $37.2(103)$ & $14.3(22)$ & $n . s$. \\
Desinhibirme & $63.2(55)^{*}$ & $63.8(208)^{*}$ & $40.8(113)^{*}$ & $18.6(29)^{*}$ & $e, b>p>n$ \\
Ligar & $57.5(50)^{*}$ & $57.4(187)^{*}$ & $38.6(107)^{*}$ & $15.6(24)^{*}$ & $e, b>p>n$ \\
Sentir euforia & $66.7(58)^{*}$ & $62.9(205)^{*}$ & $35.0(97)^{*}$ & $15.6(24)^{*}$ & $e, b>p>n$ \\
Sentirme más fuerte & $56.3(49)^{*}$ & $57.7(188)^{*}$ & $35.7(99)^{*}$ & $14.9(23)^{*}$ & $e, b>p>n$ \\
Por curiosidad & $72.4(63)^{*}$ & $79.4(258)^{*}$ & $65.7(182)^{*}$ & $26.6(41)^{*}$ & $e, b, p>n$ \\
\hline
\end{tabular}

$p<.0001$

$e=$ en exceso, $\underline{b}=$ bastante/con moderación, $p=$ poco, $d=$ nada

Fuente: elaboración propia. 
A ello se suman diversas consideraciones concernientes a la explicitación de las consecuencias negativas derivadas del consumo de alcohol para los adolescentes encuestados. En relación con las consecuencias físicas, psicológicas y sociales derivadas del consumo abusivo de alcohol, los adolescentes que abusan del alcohol informan de haber sufrido en mayor medida accidentes (18.4 $\%)$, otro problema que requirió atención médica (16.1\%), detención por la Policía (17.2 \%) y absentismo escolar (35.6\%). Igualmente, casi la mitad de la muestra de adolescentes que consumen excesivamente declaran haber participado en una riña, discusión u otro conflicto sin agresión (47.1 \%) y un $37.9 \%$ en una pelea o agresión física. En todos los indicadores explorados se ha confirmado la significación de las diferencias $(p<.0001)$ en relación con el resto de tipo de bebedores (con- sumo con moderación, poco) y adolescentes abstemios.

Por último, se propone un modelo explicativo del Uso/Abuso percibido de alcohol en adolescentes evaluado mediante un autodiagnóstico de consumo (consumo excesivo, bastante/moderación, poco/ocasional, abstinencia), de acuerdo con la capacidad explicativa de las variables exploradas (motivadores de consumo, experimentación de otras sustancias psicoactivas junto al alcohol, intoxicaciones etílicas, percepciones de riesgo, consecuencias negativas derivadas de la experimentación, etc.). Tal como se expone en las correspondientes Tablas 6 y 7 se han seleccionado trece factores/variables propuestos como los más significativos, siendo la correlación múltiple entre el criterio y las variables independientes elevada $(R$ $=.710$ ), de modo que las variables seleccionadas

TABla 5

Percepciones de riesgo y problemas asociados al uso/abuso. Diferencias por tipos de bebedores

\begin{tabular}{|c|c|c|c|c|c|}
\hline \multirow{2}{*}{ Percepción de Riesgo } & Bebedores en Exceso & Bastante/Moderación & Poco & Nada & Tukey-B \\
\hline & $\%(n)$ & $\%(n)$ & $\%(n)$ & $\%(n)$ & \\
\hline Fumar un paquete de tabaco diario & $73,6(64)$ & $83,4(272)$ & $85,8(237)$ & $83,8(129) * *$ & $n>p>b>e$ \\
\hline Tomarse 5 ó 6 copas/cañas día & $69.0(60)$ & $81,3(265)$ & $83,1(230)$ & $85,1(131) * *$ & $n, p>b>e$ \\
\hline $\begin{array}{l}\text { Fumar hachís/marihuana habitual- } \\
\text { mente }\end{array}$ & $70.1(61)$ & $81.6(266)$ & $81.1(222)$ & $82.5(127)^{* *}$ & $n, p, b>e$ \\
\hline $\begin{array}{l}\text { Tomar tranquilizantes/pastillas } \\
\text { para dormir alguna vez } \\
\text { Tomar tranquilizantes/ pastillas }\end{array}$ & $66.7(58)$ & $67.2(219)$ & $70.4(195)$ & $78.6(121)$ & n.s. \\
\hline habitualmente & $74 ., 7(65)$ & $80.7(263)$ & $81.1(222)$ & $81.8(125)$ & n.s. \\
\hline Consumir éxtasis alguna vez & $72.4(63)$ & $78.2(270)$ & $81.1(222)$ & $82.2(126)$ & $n, p>b>e$ \\
\hline Consumir éxtasis habitualmente & $77.0(67)$ & $81.8(270)$ & $82.7(229)$ & $82.5(127)$ & n.s. \\
\hline Consumir cocaína alguna vez & $69.0(60)$ & $76.4(249)$ & $80.0(221)$ & $82.5(127)$ & $n>p>b>e$ \\
\hline Consumir cocaína habitualmente & $74.7(65)$ & $77.9(254)$ & $81.6(226)$ & $84.2(130)$ & n.s. \\
\hline Problemas asociados & $\%(n)$ & $\%(n)$ & $\%(n)$ & $\%(n)$ & \\
\hline Accidente & $18.4(16)^{*}$ & $9.2(30)$ & $6.5(18)$ & $1.3(2)$ & $e>b>p>n$ \\
\hline Otro problema con atención médica & $16.1(14)^{*}$ & $5.5(18)$ & $2.9(8)$ & $1.3(2)$ & $e>b>p>n$ \\
\hline Detención por Policía & $17.2(15)^{*}$ & $4.6(15)$ & $1.8(5)$ & $0.6(1)$ & $e>b>p>n$ \\
\hline Faltar al colegio & $35.6(21) *$ & $15.3(50)$ & $5.4(15)$ & $1.9(3)$ & $e>b>p>n$ \\
\hline $\begin{array}{l}\text { Riña, discusión u otro conflicto sin } \\
\text { agresión }\end{array}$ & $47.1(41)^{*}$ & $33.1(108)$ & $14.4(40)$ & $1.9(3)$ & $e>b>p>n$ \\
\hline Pelea o agresión física & $37.9(33) *$ & $23.9(78)$ & $10.8(30)$ & $2.6(5)$ & $e>b>p>n$ \\
\hline
\end{tabular}

Niveles de respuesta: I/A/MA * $p<.0001, * * p<.05$

$e=$ en exceso, $b=$ bastante/con moderación, $p=$ poco, $d=$ nada

Fuente: elaboración propia. 
explican más del cincuenta por ciento (50.14\%) de la varianza criterio. Se comprueba que la experimentación abusiva con alcohol será más elevada cuanta menor sea la percepción de riesgo asociada (mentalidad del usuario) y mayores sean las consecuencias físicas (atención médica) y propias de conducta disruptiva (transgresión de normas, absentismo escolar, violencia física, etc.) derivadas, vinculada a motivadores deshinbitorios y con sustrato clínico, ligada a prácticas de "botellón” y asociada a experimentaciones de otras sustancias psicoactivas con especial valor discriminativo del consumo de cocaína.

TABLA 6

Variables explicativas del Uso/Abuso percibido de alcohol (Análisis de Regresión Múltiple)· Resumen del Modelo

\begin{tabular}{cccccccc}
\hline Modelo & $\mathbf{R}$ & $\mathbf{R}^{2}$ & $\mathbf{R}^{2}$ corregida & $\begin{array}{c}\text { Error típ. de } \\
\text { la estimación }\end{array}$ & $\begin{array}{c}\text { Cambio en } \\
\mathbf{R}^{2}\end{array}$ & Cambio en F & $\begin{array}{c}\text { Sig. cambio } \\
\text { en } \mathrm{F}\end{array}$ \\
\hline 1 & .710 (a) & .503 & .491 & .642 & .534 & 31.193 & .000 \\
Modelo & & & $\begin{array}{c}\text { Suma de } \\
\text { Cuadrados }\end{array}$ & $g l$ & $\begin{array}{c}\text { Media cua- } \\
\text { drática }\end{array}$ & F & Sig. \\
1 & & Regresión & 342.680 & 13 & 16.318 & 39.534 & .000 \\
& Residual & 338.055 & 819 & .413 & & \\
& Total & 680.735 & 840 & & & \\
\hline
\end{tabular}

a) Variables predictoras: (Constante), percepción de riesgo, consumo de alcohol en discotecas, mi tiempo libre lo paso solo, consumo de cocaína, consumo de alcohol fuera de casa yo solo, motivadores del primer consumo de alcohol, consecuencias del consumo abusivo de alcohol, consumo de tabaco, consumo de drogas de síntesis, consumo de alcohol en la calle con amigos ("botellón"), consumo de alcohol en casa yo solo, intoxicaciones etílicas en el último mes, consumo de hachís o marihuana. Variable dependiente: Uso/Abuso percibido: “¿Cuánto dirías que bebes?”.

Fuente: elaboración propia.

TABLA 7

Variables explicativas del Uso/Abuso percibido de alcohol (Análisis de Regresión Múltiple) C Coeficientes en la Ecuación Final.

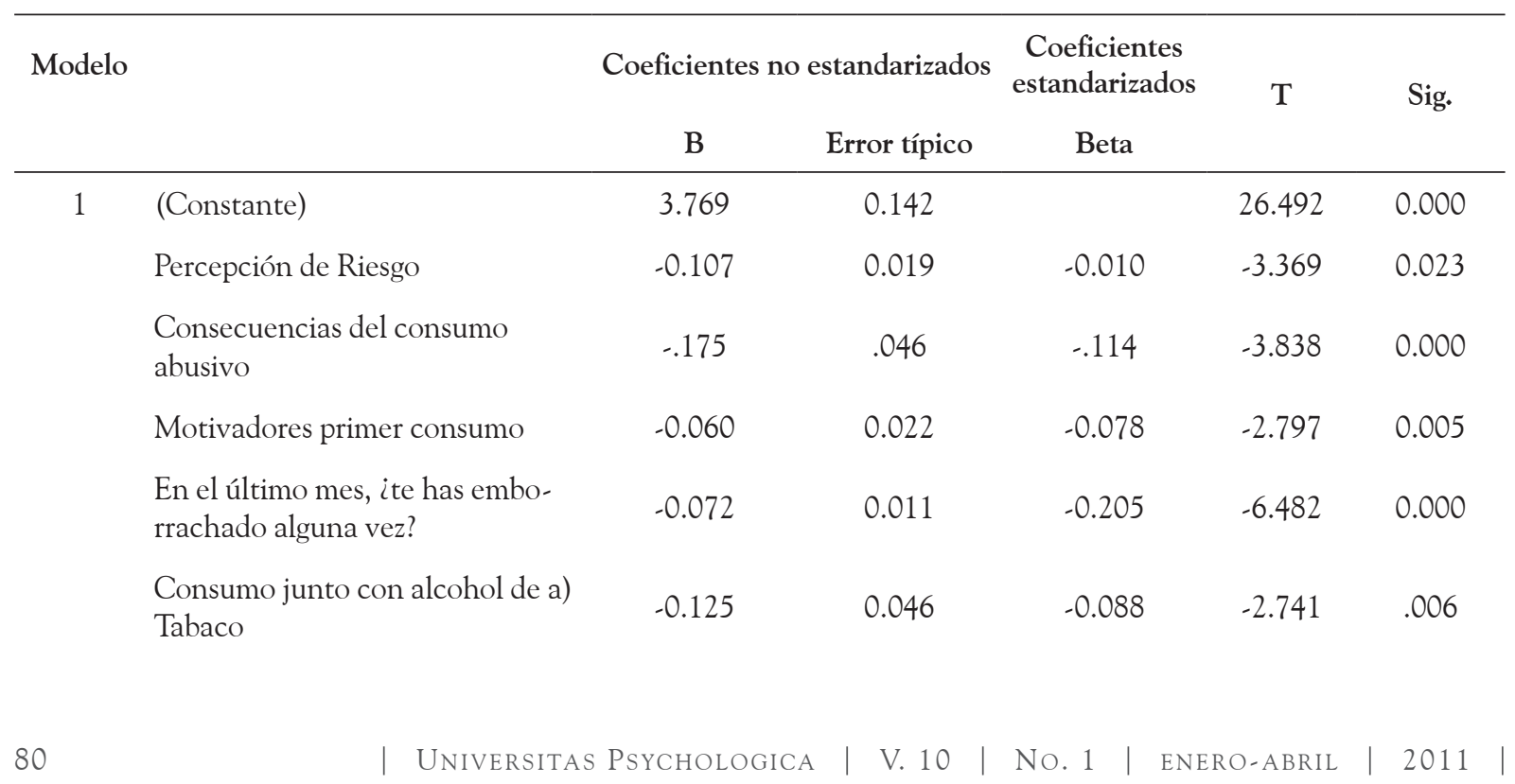




\begin{tabular}{|c|c|c|c|c|c|}
\hline \multirow[t]{2}{*}{ Modelo } & \multicolumn{2}{|c|}{ Coeficientes no estandarizados } & \multirow{2}{*}{$\begin{array}{c}\text { Coeficientes } \\
\text { estandarizados } \\
\text { Beta }\end{array}$} & \multirow{2}{*}{$\mathrm{T}$} & \multirow{2}{*}{ Sig. } \\
\hline & B & Error típico & & & \\
\hline b) Hachís o marihuana & -0.437 & 0.070 & -0.216 & -6.232 & 0.000 \\
\hline c) Drogas de síntesis & -0.121 & 0.102 & -0.037 & -2.179 & .023 \\
\hline d) Cocaína & 0.335 & 0.164 & 0.061 & 2.049 & 0.041 \\
\hline Mi tiempo libre lo paso: a) Solo & 0.029 & 0.023 & 0.034 & 1.280 & 0.041 \\
\hline $\begin{array}{l}\text { ¿Dónde has consumido alcohol } \\
\text { en los últimos } 30 \text { días?: a) En } \\
\text { casa, yo sólo }\end{array}$ & 0.016 & 0.038 & 0.012 & 0.409 & 0.683 \\
\hline b) Fuera de casa, yo solo & 0.007 & 0.036 & 0.006 & 0.198 & 0.843 \\
\hline $\begin{array}{l}\text { g) En la calle con amigos u otra } \\
\text { gente ("botellón") }\end{array}$ & -0.091 & 0.020 & -0.140 & -4.627 & 0.000 \\
\hline h) En discotecas & -0.109 & 0.019 & -0.175 & -5.845 & 0.000 \\
\hline
\end{tabular}

Variable dependiente: Uso/Abuso percibido de Alcohol: "¿Cuánto dirías que bebes?"

Fuente: elaboración propia.

\section{Discusión y Conclusiones}

El consumo juvenil de alcohol representa, en España, un problema y un reto sociosanitario de primera magnitud, máxime durante la adolescencia, a causa de las complicaciones metabólicas de las ingestas abusivas y por las consecuencias e implicaciones psicosociales de índole identitario y relacional. En el arco mediterráneo, y específicamente entre los jóvenes españoles, tal como se ha expuesto en la fundamentación teórica, se ha descrito una tendencia a la experimentación temprana con alcohol, acorde a unas pautas socioculturales permisivas ligadas a formas sobreactivadas de diversión juvenil y rituales grupales descritos bajo el calificativo "botellón" u otras modalidades en las que priman los motivadores de contacto social y desinhibitorios (modelo latino socializante), tendiendo a generalizarse un particular estilo de interacción y vinculación grupal asociado a unas prácticas ritualizadas lúdicas focalizadas en el fin de semana, según el morfotipo de consumo anglosajón (Aguilera, 2002; Calafat et al., 2004; Calafat, 2007; Cortés et al., 2008; Franco et al., 2005; Gómez-Fraguela et al., 2008), lo cual ha sido confirmado en este estudio.
Concretamente, de acuerdo con las aportaciones específicas de este trabajo a esta cuestión, se incide en los siguientes aspectos:

a) En su conjunto, se confirma la manifestación de un estilo de esparcimiento juvenil con coincidencia en actividades identificativas de sus prácticas lúdicas con un motivador eminentemente relacional. Estos resultados concuerdan con otros en los que se vinculan semejantes usos al grupo de amigos como hábito compartido, actuando incluso como mecanismo de vinculación e integración grupal (Kloep, Hendry, Ingebrigtsen, Glendinning \& Espnes, 2001). Se prioriza el consumo zocal (Moral, 2007) asociado a contextos lúdicos y escenarios sociales (bares, pubs, calle, etc.) en compañía de amigos u otra gente, lo cual se asocia a ciertas motivaciones psicosociales de consumo.

b) Se ha evaluado la implicación de una variable moduladora de la experimentación, como es el nivel de edad. En función del mismo, se han hallado diferencias relativas al hábito social de consumo de alcohol. Baste recordar que, según la literatura especializada, los adolescentes y 
preadolescentes (siendo los doce-trece años las edades críticas para el primer consumo) representan el colectivo con mayor riesgo de experimentación de drogas institucionalizadas (Delegación del Gobierno para el Plan Nacional sobre Drogas, 2007). Tales resultados son similares a otros hallados en otros estudios con adolescentes latinoamericanos, en los que se incide en que el consumo alcohol es prevalente entre los jóvenes, con grandes similitudes en el promedio de edad de inicio al consumo (Alonso, Pérez, Alcalá, Lubo \& Consuegra, 2008; Lale-Demoz \& Cumsille, 2006).

c) Se han obtenido resultados diferenciales en motivadores de consumo, percepción de riesgo y consecuencias derivadas del tipo de consumo en función de la percepción del tipo de bebedor (excesivo a abstemio), en el sentido predicho vinculado al proceso de mentalidad del usuario descrito. Así, la asociación entre beber y prácticas de conductas de riesgo va en la misma dirección que los resultados obtenidos por Calafat, Blay et al. (2009).

d) Específicamente, en relación con el tipo de motivadores para el consumo, destacan los de carácter desinhibitorio, confirmándose la existencia de diferencias significativas atribuibles al perfil tipo de bebedor, así como los relativos al hallazgo de mayor sustrato clínico en la primera experimentación en adolescentes que consumen abusivamente. De este modo, se confirma el motivador relacional de consumo con mecanismos de identificación grupal, siendo mínimos los porcentajes adscritos al morfotipo de joven consumidor en soledad con tendencia toxicofólica y mayores desajustes psicopatológicos y relacionales (Sirvent et al., 2007).

e) Se ha confirmado la tendencia descrita a una percepción de riesgo distorsionada, acorde con los resultados obtenidos por Castellana y Lladó (1999), entre otros. De este modo, las opiniones de los adolescentes en riesgo percibido (Bebedores excesivos) son indicativas de una mayor permisividad ante ciertos usos/abusos de alcohol, de una inclinación más favorable a la experimentación y de una disposición para el consumo más permisiva. De este modo, se ha comprobado la significación de las diferencias atribuibles al tipo de bebedor en los riesgos percibidos en la dependencia tabáquica, la habituación al alcohol, el consumo habitual de derivados canábicos y el consumo experimental de éxtasis y cocaína.

f) En el aspecto relativo a las consecuencias negativas de índole físico (atención médica), delictivo (detención por la Policía) y psicosocial (violencia interpersonal), derivadas del consumo de alcohol, se han obtenido diferencias significativas entre los adolescentes que declaran consumir abusivamente y el resto de bebedores ocasionales y abstemios. De especial significación psicosocial es el indicador relativo a las implicaciones en agresiones físicas, que ha de interpretarse vinculando los efectos anestésicos del alcohol al mecanismo de decorticación cerebral, dándose rienda suelta a la tensión agresiva, y a variables más psicosociales que condicionan la falta de audacia para evacuar adecuadamente dicha tensión.

g) Por último, se ha ofrecido un modelo explicativo del Uso/Abuso percibido de alcohol en adolescentes evaluado mediante un autodiagnóstico de consumo. Se ha comprobado la mediación explicativa de la percepción de riesgo asociada (mentalidad del usuario), de las consecuencias derivadas de la experimentación, la acción de motivadores deshinbitorios y con sustrato clínico, y la acción determinante de la experimentación con otras drogas (especialmente se ha confirmado el valor discriminativo del consumo de cocaína). Tales resultados van en la misma línea que otros en los que se ofrece un modelo explicativo de los patrones de consumo de alcohol en la adolescencia, atendiendo a la percepción de peligrosidad (véanse Cortés et al., 2008; Moral \& Ovejero, 2003, 2009; Salamó et al., 2010).

Como contribución significativa, original y pertinente del estudio los autores consideramos que, aparte del acuerdo en considerar esta problemática como un reto sociosanitario de primera magnitud 
tanto en el arco mediterráneo como en Latinoamérica lo cual justificaría la difusión internacional de estudios epidemiológicos y descriptivos, la contribución singular de este estudio es ofrecer un análisis de los motivadores de consumo, de las percepciones de riesgo y de los problemas asociados en función del autodiagnóstico del uso/abuso de alcohol reportado por los propios adolescentes. Asimismo, se ofrece un modelo explicativo del uso/abuso percibido de alcohol mediante el que se comprueba la percepción de riesgo asociada (mentalidad del usuario), la implicación de motivadores vinculados al deseo de desinhibición y que concurren con experimentaciones de otras sustancias psicoactivas (entre ellas cannabis y cocaína). Como limitaciones del estudio se incide en las propias del carácter transversal del estudio, así como las derivadas de la selección muestral no aleatoria y en el empleo de medidas autoinformadas (sesgo de deseabilidad social), que se intentaron contrarrestar mediante la garantía del anonimato y la confidencialidad de los datos

Como líneas prospectivas de actuación, se incide en la necesidad de profundizar en los análisis comprehensivos de esta problemática, de cara al diseño e implementación de programas de prevención e intervención, tanto en el ámbito educativo, como familiar y comunitario, con políticas que superen localismos e integren mecanismos de intervención colaborativa entre agencias de socialización e instituciones nacionales e internacionales acorde a políticas preventivas de carácter integrado tales como el Plan Europeo de actuación sobre el alcohol (Flores, González, Robledo, Rubio, Espiga \& Sáiz, 2000; Institute on Alcohol Studies, 2006) o los programas específicos de actuación de la Organización Panamericana de la Salud (2003, 2008), en virtud de los cuales se atienda al estado global de la cuestión (WHO, 2004, 2005, 2007, 2008). También convendría incrementar la publicación de estudios de revisión de las estrategias y políticas de prevención, tales como los emprendidos por el European Monitoring Centre for Drugs and Drug Addiction (EMCDDA, 2006, 2009) a nivel europeo y a través de la European Institute of Studies on Prevention con revisiones como las de Calafat,
Juan y Duch (2009) en España, los trabajos desarrollados por Faggiano y Vigna-Taglianti (2008) en Estados Unidos, así como estudios sobre los impactos de las políticas públicas como el emprendido por la Comisión Latinoamericana sobre Drogas y Democracia (2009) en el contexto latinoamericano. Por último, dado el reto sociosaniatrio que representa esta problemática, se aboga por el diseño de estrategias conjuntas de actuación como las propuestas por la Estrategia Nacional sobre Drogas 2009-2016 (Delegación del Gobierno para el Plan Nacional sobre Drogas, 2009) y el incremento de los esfuerzos en el tratamiento integral y global de esta problemática mediante la promoción conjunta de la UE y Latinoamérica a través de agencias como la Comisión Iberoamericana para el Control del Abuso de Drogas de la Organización de Estados Americanos (CICAD) en la prevención y el tratamiento del uso y la dependencia de drogas.

\section{Referencias}

Aguilera, R. (2002). Generación botellón. Madrid: Oyeron.

Alonso, L. M., Pérez, M. A., Alcalá, G., Lubo, A. \& Consuegra, A. (2008). Comportamientos de riesgo para la salud en estudiantes colombianos recién ingresados a una universidad privada en Barranquilla (Colombia). Salud Barranquilla, 24(2), 235-247.

Ayesta, F. J. (2002). Bases bioquímicas y neurobiológicas de la adicción al alcohol. Adicciones, 14 (Supl. 1), 63-79.

Bach i Bach, L. (2000). La falta de conciencia del daño alcohólico, firme impedimento para la prevención primaria y secundaria del alcoholismo. Revista Española de Drogodependencias, 25 (2), 114-117.

Bellis, M. A. \& Hughes, K. (2003). Consumo recreativo de drogas y reducción de daños en la vida nocturna global. Adicciones, 15 (Supl. 2), 289-309.

Bilbao, A. (2008). Individuo y orden social. La emergencia del individuo y la transición a la sociología [Reseña]. Reis, Revista Española de Investigaciones Sociológicas, 123, 251-255.

Bobes, J., Bascarán, M. T., González, M. P. \& Sáiz, P. A. (2000). Epidemiología del uso/abuso del cannabis. Adicciones, 12 (Supl. 2), 31-40. 
Bouzada, X. (2001). Los espacios del consumo cultural colectivo. Reis, Revista Española de Investigaciones Sociológicas, 96, 51-70.

Brändle, G. (2007). Consumo y cambio social en España: evolución en el equipamiento doméstico 1983-2005. Reis, Revista Española de Investigaciones Sociológicas, 120, 75-114.

Bravo, O. A. (2007). Análisis de los discursos sobre drogas en las Instituciones Públicas del Distrito Federal Brasil. Psicología desde El Caribe, 20, 188-207.

Cáceres, D., Salazar, I., Varela, M. \& Tovar, J. (2006). Consumo de drogas en jóvenes universitarios y su relación de riesgo y protección con los factores psicosociales. Universitas Psychologica, 5 (3), 521-534.

Calafat, A. (2007). El abuso de alcohol de los jóvenes en España. Adicciones, 19 (3), 217-223.

Calafat, A., Blay, N., Juan, M., Adrover, D., Bellis, M. A., Hughes, K. et al. (2009). Traffic risk behaviours at nightlife: Drinking, taking drugs, driving and use of public transport by young people. Traffic Injury Prevention, 10(2), 162-169.

Calafat, A., Juan, M., Becoña, E., Castillo, A., Fernández, C., Franco, M. et al. (2004). El consumo de alcohol en la lógica del botellón. Adicciones, 17(3), 193-202.

Calafat, A., Juan, M., Becoña, E., Fernández, C., Gil, E., Palmer, A. et al. (2000). Salir de marcha y consumo de drogas. Madrid: Delegación del Gobierno para el Plan Nacional sobre Drogas.

Calafat, A., Juan, M., Becoña, E. \& Mantecón, A. (2008). ¿Qué drogas se prefieren para las relaciones sexuales en contextos recreativos? Adicciones, 20(1), 37-48.

Calafat, A., Juan, M. \& Duch, M. A. (2009). Intervenciones preventivas en contextos recreativos nocturnos: revisión. Adicciones, 21 (4), 387-414.

Casa, M. J., O’Ferrall, C. \& Vaca, F. J. (2001). Evolución del perfil del enfermo alcohólico durante los últimos veinte años. Revista Española de Drogodependencias, 26(2), 127-134.

Castellana, M. \& Lladó, M. (1999). Adolescencia y juventud: Adolescencia y juventud: prevención y percepción del riesgo al consumo. Revista Española de Drogodependencias, 24, 118-130.
Comas, D. (2004). Jóvenes y estilos de vida. Valores y riesgos en los jóvenes urbanos. Reis, Revista Española de Investigaciones Sociológicas, 104, 264-268.

Comas, R., Jiménez, A., Acero, A. \& Carballo, C. (2007). Variables psicosociales del consumo de cannabis en adolescentes. Revista Española de Drogodependencias, 2, 162-180.

Comisión Nacional de Investigación en Drogas. (2001). Encuesta Nacional sobre consumo de Sustancias psicoactivas en jóvenes de 10 a 24 años. Bogotá: Autor.

Comisión Latinoamericana sobre Drogas y Democracia. (2009). Drogas y democracia: hacia un cambio de paradigma. Recuperado el 8 de octubre de 2009, de http://www.idpc.net/sites/default/files/library/Drogas $\% 20 \mathrm{y} \% 20$ democracia $\% 20$ hacia $\% 20$ un $\% 20$ cambio\%20de\%20paradigmo.pdf.

Cortés, M. T., Espejo, B. \& Giménez, J. A. (2008). Aspectos cognitivos relacionados con la práctica del botellón. Psicothema, 20(3), 396-402.

Delegación del Gobierno para el Plan Nacional sobre Drogas. (2004). Encuesta estatal sobre uso de drogas en Enseñanzas Secundarias. Madrid: Ministerio de Sanidad y Consumo.

Delegación del Gobierno para el Plan Nacional sobre Drogas. (2007). Encuesta estatal sobre uso de drogas en Enseñanzas Secundarias. Madrid: Ministerio de Sanidad y Consumo.

Delegación del Gobierno para el Plan Nacional sobre Drogas. (2009). Estrategia Nacional sobre Drogas 2009-2016. Madrid: Ministerio de Sanidad y Política Social.

Echeburúa, E. (1985). Terapias aversivas en el tratamiento del alcoholismo: una revisión. Drogalcohol, 10(1), 27-34.

Echeburúa, E. \& Del Corral, P. (1988). Evaluación y tratamiento de un caso clínico de alcoholismo. En D. Macià \& F. X. Méndez (Eds.), Aplicaciones clínicas de la evaluación y modificación de conducta. Estudio de Casos (pp. 147-207). Madrid: Pirámide.

Espada, J. P., Pereira, J. R. \& García-Fernández, J. M. (2008). Influencia de los modelos sociales en el consumo de alcohol de los adolescentes. Psicothema, 20(4), 531-537.

European Monitoring Centre for Drugs and Drug Addiction. (2006). Developments in drug use within recreational settings. Lisbon: Author. 
European Monitoring Centre for Drugs and Drug Addiction. (2009). Trends and availability of responses to drug use in recreational settings in Europe. Poster downloadable. Recuperado el 1 de abril de 2010, de http://www.emcdda.europa.eu/attachements.cfm/att_78666_en_RES_2020.pdf

Examen Médico Automatizado para Estudiantes. (2005). Dirección General de Servicios Médicos. México: Secretaría Administrativa UNAM.

Faggiano, F. \& Vigna-Taglianti, F. (2008). Drugs, illicit - primary prevention strategies. In K. Heggenhougen \& S. Quah (Eds.), International Encyclopedia of Public Health (Vol 2., pp. 249-265). San Diego: Academic Press.

Flores, D., González, J., Robledo, T., Rubio, J., Espiga, I. \& Saiz, I. (2000). Plan europeo de actuación sobre alcohol 2000-2005. Organización Mundial de la Salud Europea. Madrid: Dirección General de Salud Pública y Consumo. Subsecretaría de Sanidad y Consumo. Ministerio de Sanidad y Consumo.

Franco, M., Juan, M., Pereriro, C., Calafat, A., Castillo, A. \& Becoña, E. (2005). El consumo de alcohol en la lógica del botellón. Adicciones, 17 (3), 193-203.

Goethals, G. R., Messick, D. M. \& Allison, S. T. (1991). The uniqueness bias: Studies of constructive social comparison. En J. Suls \& T. A. Wills (Eds.), Social comparison: Contemporary theory and research (pp. 149-176). Hillsdale, NJ: Erlbaum.

Gómez, M. T. \& Álvarez, F. J. (2006). Jóvenes con consumo de alcohol y cannabis y accidentes e tráfico. Trastornos Adictivos, 8(1), 14-21.

Gómez-Fraguela, J., Fernández, N., Romero, E. \& Luengo, A. (2008). El botellón y el consumo de alcohol y otras drogas en la juventud. Psicothema, 20(2), 211-217.

Gual, A. (2006). Alcohol in Spain: Is it different? Addiction, $101(8), 1073-1077$.

Herrera-Vázquez, M., Wagner, F. A., Velasco-Mondragón, E., Borges, G. \& Lazcano-Ponce. E. (2004). Inicio en el consumo de alcohol y tabaco y transición a otras drogas en estudiantes de Morelos, México. Salud Pública de México, 46(2), 132-140. Igartua, J. (1996). Psicología de la Publicidad. San Sebastián: Ibaeta Psicología.

Infante, C., Barrio, G. \& Martín, E. (2003). Tendencias, características y problemas asociados al consu- mo de drogas recreativas en España. Adicciones, 15(2), 77-96.

Institute on Alcohol Studies. (2006). El alcohol en Europa: una perspectiva de Salud Pública. Bruselas: Comisión Europea.

Kandel, D. B. (1975). Stages in adolescent involvement in drug use. Science, 190, 912-914.

Kandel, D. B. (1978). Longitudinal research on drug use. Empirical findings and methological issues. Washington, D.C.: Wiley.

Kloep, M., Hendry, L. B. L., Ingebrigtsen, J. E., Glendinning, A. \& Espnes, G. A. (2001). Young people in 'drinking' societies? Norwegian, Scottish and Swedish adolescents'perception of alcohol use. Health Education Research, 16(3), 279-291.

Krueger, J. \& Clement, R. W. (1994). The truly false consensus effect: An ineradicable and egocentric bias in social perception. Journal of Personality and Social Psychology, 67, 596-610.

Lale-Demoz, A. \& Cumsille, F. (Coords.). (2006). Jóvenes y drogas en países sudamericanos: un desafío para las políticas públicas. Primer estudio comparativo sobre uso de drogas en población escolar secundaria de Argentina, Bolivia, Brasil, Colombia, Chile, Ecuador, Paraguay, Perú y Uruguay. Sistema Subregional de Información e Investigación sobre Drogas.

Londoño, C. \& Valencia, C. (2008). Asertividad, resistencia a la presión de grupo y consumo de alcohol en universitarios. Actas Colombianas de Psicología, $11(1), 155-162$.

Lucas, A. C. S., Parente, N. S., Picanço, D. A., Conceição, K. R. C., Magalhães, I. R. S. \& Siqueira, J. C. A. (2005). Uso de drogas entre universitarios de la Facultad de Ciencias de la Salud de UFAM Brasil. Revista de Toxicología, 22(2), 126-128.

Marks, G. \& Miller, N. (1987). Ten years of research on the false-consensus effect: An empirical and theoretical review. Psychological Bulletin, 102, 72-90.

Ministerio de Educación, República de Colombia. (2006). El $80 \%$ de los estudiantes de Colombia consume alcohol y el $48 \%$, tabaco. Recuperado el 10 de diciembre de 2008, de http: //www.mineeducacion.gov.co/cvn /1665/fo-article-98340.pdf

Ministerio de Protección Social, República de Colombia. (2003). El Estudio Nacional de Salud Mental 
Colombia 2003. Recuperado el 10 de diciembre de 2008, de http:/www.abacolombia.org.co/bv/ clinica/estudio_salud_mental_colombia.pdf_

Moral, M. V. (2007). Anecdotario de la cultura de la droga: psicofármacos domésticos como elixires de antaño y psicoestimulantes de hoy. Encuentros en Psicología Social, 4, 23-33.

Moral, M. V. \& Ovejero, A. (2003). Actitudes ante el consumo de sustancias psicoactivas y mentalidades del usuario en adolescentes de Secundaria. Entemu. Revista de la UNED Centro Asociado de Asturias, 15, 151-175.

Moral, M. V. \& Ovejero, A. (2006). Ocio dionisíaco y experimentación con sustancias psicoactivas: aproximación crítica desde la Psicología Social. Revista de Psicología General y Aplicada, 59(1-2), 241-255.

Moral, M. V. \& Ovejero, A. (2009). Experimentación con sustancias psicoactivas en adolescentes españoles: perfil de consumo en función de los niveles de edad. Revista Latinoamericana de Psicología, 41(3), 533-554.

Moral, M. V., Rodríguez, F. J. \& Sirvent, C. (2005). Motivadores de consumo de alcohol en adolescentes: análisis de las diferencias intergénero y propuesta de un continuum etiológico. Adicciones, $17(2), 105-120$.

Observatorio Español sobre Drogas. (2002). Encuesta escolar sobre consumo de drogas 2002. Madrid: Ministerio de Sanidad y Consumo.

Observatorio Español sobre Drogas. (2004). Informe 2004. Situación y tendencias de los problemas de drogas en España. Madrid: Ministerio de Sanidad y Consumo.

Oficina contra la droga y el crimen de la Organización de las Naciones Unidas. (2004). World Drug Report 2004. Recuperado el 9 de abril de 2010, de http://www.unodc.org/unodc/en/world_drug_report_2004.html

Olivar, A. \& Carrero, V. (2007). Análisis de la intención conductual de consumir cánnabis en adolescentes: desarrollo de un instrumento de medida basado en la teoría de la conducta planificada. Trastornos Adictivos, 9(3), 184-205.

Organización Panamericana de la Salud. (2003). Reporte reunión del Comité Asesor de Investigación en Salud.
Recuperado el 10 de diciembre de 2008, de http:// www.paho.org

Organización Panamericana de la Salud. (2005). Encuesta de salud de escolares del Caribe: urge reforzar los factores protectores. Revista Panamericana de Salud Pública, 17 (1), 48-50.

Organización Panamericana de la Salud. (2008). Reporte Directora OPS. La planificación de acciones de salud pública. Una visión colectiva y un compromiso para lograr una salud mejor. Recuperado el 2 de enero de 2009, de http: //www.paho.org

Rodríguez, J., Valdés, M., Ramírez, S., Hernández, E., Villalón, M. \& Fernández, A. M. (2008). Factores predictores del riesgo de consumo de drogas lícitas en escolares de 4ํㅜ a 7oㅡ básico. Terapia Psicológica, 26(2), 181-188.

Rodríguez-Martos, A.(1996). Factores de riesgo. Prevención. Detección e intervención en problemas de alcohol en la población infanto-juvenil. En E. Gil, T. Robledo, J. Rubio \& I. Espiga (Eds.), Alcohol y Juventud 1995 (pp. 61-86). Madrid: Ministerio de Sanidad y Consumo, Centro de Publicaciones de la Secretaría General Técnica.

Sáiz, P. A., González, M. P., Paredes, B., Delgado, J. M., López, J. L., Martínez, S. \& Bobes, J. (2001). Consumo de MMDA (éxtasis) en estudiantes de secundaria, Adicciones, 13 (2), 159-171.

Salamó, A., Gras, M. E. \& Font-Mayolas, S. (2010). Patrones de consumo de alcohol en la adolescencia. Psicothema, 22 (2), 189-195.

Salazar, I. C. \& Arrivillaga, M. (2004). El consumo de alcohol, tabaco y otras drogas como parte del estilo de vida de los jóvenes universitarios. Revista Colombiana de Psicología, 13, 74-89.

Sanders, E. (Ed.). (2006). Drugs, clubs and young people: Sociological and public health perspective. Burlington: Ashgate.

Sirvent C., Moral M. V. \& Rodríguez, F. J. (2007). Jóvenes $\mathcal{E}$ Alcohol. Conjunto Didáctico-Audiovisual para Educadores, Psicólogos y Colectivos Mediadores. Valencia: Nau Llibres.

Torres, M. C., Palma, M. M., Iannini, J. \& Moreno, S. (2006). Validación de la prueba Young Adult Alcohol Problems Screening Test, YAAPST, en un grupo de estudiantes universitarios de la Pontificia 
Universidad Javeriana de Bogotá. Universitas Psychologica, 5(1), 175-190.

Urquieta, J. E., Hernández-Ávila, M. \& Hernández, B. (2006). El consumo de tabaco y alcohol en jóvenes de zonas urbanas marginadas de México: un análisis de decisiones relacionadas. Salud Pública de México, 48 (Supl.1), 30-40.

Valbuena, F., Aladro, E. \& Sáez, R. (2007). Motivaciones de los jóvenes para beber en grupo y consecuencias personales, sociales y medioambientales. Revista Española de Drogodependencias, 4, 531-543.

Vázquez, F. \& Becoña, E. (2000). Factores de riesgo y escalada cannabinoide. Adicciones, 12 (2), 175-184.

Vega, A. (2002). La sombra del botellón es alargada. Revista Española de Drogodependencias, 4, 603-617.

World Health Organization. (2004). Global status report on Alcohol, 2004. Recuperado el 21 de enero de 2009, de http: //www.who.int/substance_abuse/ publications/alcohol/en/index.html

World Health Organization. (2005). Problemas de salud pública causados por el uso nocivo del alcohol (Informe de la Secretaría en la 58 a asamblea mundial de la salud). Recuperado el 9 de abril de 2010, de http://www.who.int/substance_abuse/report_by_ secretariat_wha_58_public_health_problems_alcohol_spanish.pdf

World Health Organization. (2007). WHO expert Committee on problems related to alcohol consumption. Recuperado el 21 de enero de 2009, de http:// www.who.int/substance_abuse/expert_committee/en/index.html

World Health Organization. (2008). The World Health Report 2008. Primary Health Care: Now More Than Ever. Recuperado el 21 de enero de 2009, de http:// www.who.int/substance_abuse/publications/alcohol/en/index.html

Yubero, S., Larrañaga, E., Navarro, R., Serna, C. \& Martínez, I. (2005). La percepción del consumo de alcohol: el análisis de los sesgos atributivos como orientación para la intervención social con jóvenes. Boletín Informativo de Trabajo Social, 8, 11-18. 
